\title{
Magnetic interactions in the energy operator matrix of configurations with $s$ and $i$ electrons in external shells
}

\author{
Galina Pavlovna Anisimova ${ }^{1}$, Olga Aleksandrovna Dolmatova ${ }^{1, ~ *, ~ A n n a ~ P e t r o v n a ~ G o r b e n k o ~}{ }^{1}$, \\ Martin Tchoffo ${ }^{2}$
}

${ }^{1}$ Saint -Petersburg State University, Faculty of Physics, 198504, Stary Peterhof, Saint-Petersburg, Russian Federation

${ }^{2}$ Department of Physics, Faculty of Science, University of Dschang, Po Dschang, Cameroon

\section{Email address:}

olgadolmatova@gmail.com (O. A. Dolmatova),mtchoffo2000@yahoo.fr (M. Tchoffo)

\section{To cite this article:}

Galina Pavlovna Anisimova, Olga Aleksandrovna Dolmatova, Anna Petrovna Gorbenko, Martin Tchoffo. Magnetic Interactions in the Energy Operator Matrix of Configurations with $S$ and $i$ Electrons in External Shells. American Journal of Modern Physics. Vol. 2, No. 6, 2013, pp. 334-340. doi: 10.11648/j.ajmp.20130206.20

\begin{abstract}
In the energy operator matrix of four-level configurations with $S$ and $i$-electrons in external shells, we take into account the following magnetic interactions: spin-other-orbit, spin-spin, and orbit-orbit interactions. The calculation of matrix elements is done in the uncoupled moments representation with subsequent translation (passage) to the LSJMrepresentation. Comparison with independent calculations in LSJM-representation for $S i$ and $i s$ configurations is done. The method of calculation in electronic configuration is extended to the holes configurations $i^{25} S$. As results the energy operator matrix is complemented with new physical conditions on radial integrals of Marvin's spin interactions, which permits to ameliorate the precision of future calculations of fine structure parameters by semi-empirical methods.
\end{abstract}

Keywords: Energy Operator Matrix, LSJM Representation, Spin-Other-Orbit and Spin-Spin Magnetic Interactions, Uncoupled Moments Representation, Orbit-Orbit Interaction

\section{Introduction}

The higher excited $n s n^{\prime} i$ configurations are practically not investigated. They are realized in the Helium atom, group II atoms of the periodic table, their ions and their isoelectronic series (for instance, $\mathrm{Mg}$ I, Cd I, In II, etc..). Up to now there exist only experimental energy levels of fine structure of four 1 sni $(\mathrm{i}=7-10)$ Helium atom configurations [1]. Therefore the problem of construction of the energy operator matrix of these systems, taking into account the maximum possible number of interactions in the Breit's Hamiltonian, in particular with small magnitude spin-other-orbit and spin-spin magnetic interactions as well as orbit-orbit interaction is still unsolved. The main attention will be given to these interactions in this paper.

\section{Method of Calculation}

The calculation of the angular coefficients in the radial integrals is done in one configuration, in the formalism of irreducible tensor operators and in two representations, namely the uncoupled moments and LSJM using Lithuanian's monograph formulae of general form as in [2].
The uncoupled moments representation (used by authors of [2]) is the most adequate because in it all calculations lead basically to calculation of $3 \mathrm{j}$ Wigner symbols (but not to $9 \mathrm{j}$-symbols, like in LSJM-representation). In the present paper one considers again the nin's configuration (they are realized with elements of the $4^{\text {th }}$ group of the periodic table) and holes $n i^{25} n$ 'sconfigurations (rare gas atoms, except helium). In the uncoupled moments representation the energy operator is differentiated by the magnetic quantum number $M$ (the same as in the external electric and magnetic fields). Furthermore, in this representation the state of two electron atoms (i.e. two electrons of the external shell, moving in the central symmetric field of nuclei and electrons of full shells) depend only on the individual quantum numbers of the electrons. Thus the angular part of the wave functions in the uncoupled moments representation is given the following set of quantum numbers: $l_{1} l_{2} s_{1} s_{2} m_{l_{1}} m_{l_{2}} m_{s_{1}} m_{s_{2}}$. In the single configuration approximation the states of systems are differentiated only by the last four quantum numbers, namely the orbital and the spin projections of electrons. It is not indispensable to consider all values of $\mathrm{M}$. It is sufficient to be restricted to the energy operator matrix with $\mathrm{M}=0$, which describes all four configuration levels 
(with $M= \pm 1, \pm 2, \pm 3, \pm 4, \pm 5$ ). We recall that in the LS approximation (the LSJM representation) the configuration with $s$ and $i$ electrons have the following levels: ${ }^{3} \mathrm{I}_{7},{ }^{3} \mathrm{I}_{6},{ }^{3} \mathrm{I}_{5}$, ${ }^{1} \mathrm{I}_{6}$. Consequently, for the matrix with $\mathrm{M}=0$ (fourth level) the $s i$ wave function configuration of the independent moments representation is:

$$
\begin{array}{ccccc} 
& m_{l_{1}} & m_{l_{2}} & m_{s_{1}} & m_{s_{2}} \\
\lambda_{1} & 0 & 1 & -1 / 2 & -1 / 2 \\
\lambda_{2} & 0 & 0 & -1 / 2 & 1 / 2 \\
\lambda_{3} & 0 & -1 & 1 / 2 & 1 / 2 \\
\lambda_{4} & 0 & 0 & 1 / 2 & -1 / 2
\end{array}
$$

During the permutation of electrons in (1), the first column is interchanged with the second while the third column is swapped with the fourth. In the hole $i^{25} S$ configuration, the sign of the orbital and spin projections of $i$ electrons are changed. The change of the sign of the projections of the holes (almost full shell) helps to transform the multi-electron problem to one of two particles and permits the use of Breit's Hamiltonian.

Apart from the independent moments representation, in this paper the independent calculation of matrix elements of energy operator in the LSJM representation is done by totally different formulae than in [2]. The comparison of the results of calculation in the two representations permits exclusion of possible errors, while their concurrence justifies the accuracy of the energy operator matrix.

The transition from one representation to another is effected with the help of the matrix coefficients of Clebsch-Gordan's transformation according to the chain:

$$
l_{1} l_{2} s_{1} s_{2} L S J M \rightarrow l_{1} l_{2} s_{1} s_{2} L M_{L} S M_{S} \rightarrow l_{1} l_{2} s_{1} s_{2} m_{l 1} m_{l 2} m_{s 1} m_{s 2}
$$

The last term in (2) is the angular part of the wave function in the independent moments representation, as said earlier.

Table1. Matrix coefficients of wave functions transformation of LSJM representation through wave functions of independent moments representation for $M=0$.

\begin{tabular}{ccccc}
\hline & $\mathbf{0 1 - -}$ & $\mathbf{0 0 - +}$ & $\mathbf{0 - 1 + +}$ & $\mathbf{0 0 + -}$ \\
\hline${ }^{3} I_{7}$ & $\sqrt{3} / \sqrt{13}$ & $\sqrt{7} / \sqrt{26}$ & $\sqrt{3} / \sqrt{13}$ & $\sqrt{7} / \sqrt{26}$ \\
${ }^{3} I_{6}$ & $1 / \sqrt{2}$ & 0 & $-1 / \sqrt{2}$ & 0 \\
${ }^{3} I_{5}$ & $\sqrt{7} / \sqrt{26}$ & $-\sqrt{3} / \sqrt{13}$ & $\sqrt{7} / \sqrt{26}$ & $-\sqrt{3} / \sqrt{13}$ \\
${ }^{1} I_{6}$ & 0 & $-1 / \sqrt{2}$ & 0 & $1 / \sqrt{2}$ \\
\hline
\end{tabular}

Remark. In the heading of the table the wave functions are brought back from (1). The spin projections are denoted only by the sign. The matrix is unitary, i.e. the normalization and of the orthogonality conditions of Clebcsh-Gordan coefficients are fulfilled.

The Clebsch-Gordan's matrix coefficients for $M=0$ is presented in table1 (our calculation of the matrix coefficients for all values of $M$, are not presented here because they are bulky).

Let's point out that for further determination of the fine structure parameters by semi empirical methods, and also for the study of the Zeeman splitting one uses the energy operator matrix of LSJM representation which is the most compact in comparison with the many dimensional matrices, written in the independent moments representation.

\section{Orbit-Orbit and Electrostatic Interactions}

Even though, the calculation of matrix elements of energy operator of the electrostatic interaction in the LSJM representation does not present difficulties, angular coefficients of radial Slater's integrals for all $s l$ configurations do, see appendix 2 of [2]. Let's consider the two interactions at the same time according to the following principle: In our previous articles [3,4] it was shown that, the angular coefficients for Slater's exchange radial integrals $G_{k}$ and the set of exchange radial integrals (further denoted by Roman numbers I and II),referring to the orbit-orbit interaction, are the same as in the independent moments representation(see formulae (3) and (4) below). This reduces the calculation for exchange matrix elements of energy operator matrix of the orbit-orbit interaction a lot, (see formula (9.38b) in [2]). The cited formula contains the summation on four parameters, and also Racah's coefficients $[5,6]$, depending on these parameters. Effecting in it all the necessary mathematical operations, we obtain the following " working" formula for the calculation of exchange matrix elements of energy operator matrix for orbit-orbit interaction:

$$
\begin{aligned}
& \left|H_{12}^{00}\right|_{\text {exch. }}=(-1)^{l_{1}+l_{2}+s_{1}+s_{2}-m_{l 1}-m_{l 2}-m_{s 1}-m_{s 2}}\left(-\frac{1}{\sqrt{13}}\right) t_{12}^{000} \times \\
& \times\left(z_{12}^{000}+3 \cdot z_{12}^{110}\right) \cdot(I+I I) \cdot \delta\left(m_{s 1} m_{s 2}^{\prime}\right) \delta\left(m_{s 2} m_{s 1}^{\prime}\right)
\end{aligned}
$$

And for electrostatic interaction from (5.23b) [2] we have:

$$
\left|\frac{1}{r_{12}}\right|=-\frac{1}{\sqrt{13}} t_{12}^{000}\left(z_{12}^{000}+3 \cdot z_{12}^{110}\right) G_{6}
$$

(The phase factor and $\delta$-condition are the same, as in (3)). The stated formula is a many component expression, where $t_{12}^{x x 0}$ and $z_{12}^{x^{\prime} x^{\prime} 0}$ are the unit two-electron orbital and 
spin operator respectively. Furthermore the orbital parameter of summation $x$ takes the values: $x=0,10 \ldots, \min \left(2 l_{1}, 2 l_{2}\right)$ while the spin parameter of summation $x^{\prime}=0,1$ (see [2]). $t_{12}^{x x 0}$ and $z_{12}^{x^{\prime} x^{\prime} 0}$ are nothing but the scalar product of orbital and spin parameters of the individual electrons, i.e.

$$
t_{12}^{000}=t_{1}^{2} \cdot t_{2}^{0} ; z_{12}^{000}=z_{1}^{0} \cdot z_{2}^{0} ; z_{12}^{110}=z_{1}^{1} \cdot z_{2}^{1}
$$

Where the lower indices 1 and 2 refer to the first $(s)$ and the second (i) electron respectively.

The Wigner-Eckart theorem is applied to each unit of one-electron operators, in which the matrix elements of irreducible operators are the product of the phase factor, the matrix element and the Wigner's $3 \mathrm{j}$-symbol (the phase factor is already written in (3)).For the unit operators the stated matrix elements are equal to unity, that is why the calculation by formulae (3) and (4) leads to the calculation of $3 \mathrm{j}$-symbols.

Due to the fact that the energy operators of electrostatic interaction and the orbit-orbit interaction do not contain the spin variables and are scalar, in formulae (3) and (4) appears the $\delta$-condition on spin projection of electrons, which reduces the number of non-null matrix elements.

Table 2. Angular coefficients for radial integrals in the exchanged matrix elements of energy operator for orbit-orbit interaction.

\begin{tabular}{|c|c|c|c|c|c|c|}
\hline & $N_{k-1}$ & $K^{\prime z}$ & $K^{\prime \mu}$ & $K^{\prime \prime z}$ & Common factor for the line & Configuration \\
\hline I $k=5 K=6(k+1)$ & -4 & 1 & 1 & -1 & $42 / 11$ & \multirow[b]{2}{*}{ si } \\
\hline II $k=7 \quad K=6(k-1)$ & 4 & -1 & -1 & 1 & $14 / 5$ & \\
\hline I $k=5 K=6(k+1)$ & 120 & -6 & 5 & -1 & $42 / 11$ & \multirow{2}{*}{$\begin{array}{c}i s \\
i^{25} s\end{array}$} \\
\hline II $k=7 K=6(k-1)$ & -224 & 8 & -7 & 1 & $14 / 5$ & \\
\hline
\end{tabular}

The contents of the blocks I and II in formula (3) are presented in table 2, where $N_{k-1}$ is the Marvin's radial exchange integral [2]; $K^{\prime z}, K^{\prime u}, K^{\prime \prime}$ are radial exchange integrals, related to the Marvin's integral. The summation parameter in the exchange terms takes the values:

$$
k-1=\left|l_{1}-l_{2}\right|-2,\left|l_{1}-l_{2}\right|, \ldots, l_{1}+l_{2},
$$

i.e. for the considered configurations $k=5$ and 7. The summation parameter $K=k \pm 1$.

The calculation of the matrix elements (3) with the wave function in the independent momenta representation (1) gave the following results:

$$
\begin{array}{cc}
s i, i s & i^{25} s \\
\lambda_{1} \lambda_{1}=\lambda_{3} \lambda_{3}=-\frac{1}{13}(I+I I) & \lambda_{2} \lambda_{2}=\lambda_{4} \lambda_{4}=-\frac{1}{13}(I+I I) \\
\lambda_{2} \lambda_{4}=\lambda_{4} \lambda_{2}=-\frac{1}{13}(I+I I) & \lambda_{2} \lambda_{4}=\lambda_{4} \lambda_{2}=\frac{1}{13}(I+I I)
\end{array}
$$

The non-written matrix elements in (6) are equal to zero.

The translation of the expressions (6) from the independent momenta representation to the LSJM representation with the help of coefficients from table 1 leads to the following matrix elements:

$$
\begin{array}{cc}
\text { si,is } & i^{25} s \\
{ }^{3} I^{3} I=-\frac{1}{3}(I+I I) & { }^{3} I^{3} I=0 \\
{ }^{1} I^{1} I=\frac{1}{13}(I+I I) & { }^{1} I^{1} I=-\frac{2}{13}(I+I I)
\end{array}
$$

Opening the contents of the blocks in (7) with help of the table 2, we have the following exchange matrix elements of energy operator matrix for orbit-orbit interaction of $s i$ configurations:

$$
\begin{gathered}
{ }^{1} I^{1} I=\frac{1}{13}(I+I I)=\frac{1}{13}\left[\frac{42}{11}\left(-4 N_{k-1}+K^{\prime z}+K^{\prime u}-K^{\prime \prime z}\right)^{k=5}+\right. \\
\left.+\frac{14}{5}\left(4 N_{k-1}-K^{\prime z}-K^{\prime u}+K^{\prime z}\right)^{k=7}\right] .
\end{gathered}
$$

The diagonal triplet matrix elements ${ }^{3} I^{3} I$ in (8) have the opposite sign.

For the is configuration:

$$
\begin{gathered}
{ }^{1} I^{1} I=\frac{1}{13}\left[\frac{42}{11}\left(120 N_{k-1}-6 K^{\prime z}+5 K^{\prime u}-K^{\prime \prime z}\right)^{k=5}+\right. \\
\left.+\frac{14}{5}\left(-224 N_{k-1}+8 K^{\prime z}-7 K^{\prime u}+K^{\prime \prime z}\right)^{k=7}\right]
\end{gathered}
$$

The triplet matrix elements ${ }^{3} I^{3} I$ in (9) have opposite sign.

Finally, for the hole $i^{25} s$ configuration the only non-null singlet matrix element ${ }^{1} I^{1} I$, is opposite in sign and two times bigger than (9) (see (7)). The diagonal triplets' matrix elements ${ }^{3} I^{3} I$ are equal to zero. This result was to be expected. Although authors of the monograph [7] do not state the energy operator matrix of electrostatic interaction for configurations with s-electrons, in the text it is indicated that for hole configurations only diagonal singlet matrix elements are different from zero.

The off-diagonal matrix elements of the said operator tend to zero because of the factor in the formula (9.38a) from [2], in which the parameter of summation $k$ takes the value $k=-1$.Thus, the orbit-orbit interaction in the configurations, with $s$ and $i$ electrons is represented by 8 exchange radial integrals (4 for each of the two values of the 
parameter $k$ ). The forms of these integrals are derived in monograph [2]. Let's remark in advance, that in the semi empirical calculation of the fine structure parameters of the orbit-orbit interaction it is impossible to separate out the electrostatic interaction parameters because of the same angular coefficient in formulae (3) and (4), i.e. their linear dependence in the corresponding equations. One could have separated in expressions (8) and (9) Marvin's integral $N_{k-1}$, which is encountered also in matrix elements of energy operator of spin-other-orbit and spin-spin interactions, as it was done in $p l$ configurations $(l=1 \div 4)[3,4,8,9]$. However for all configurations with $s$-electrons the angular coefficients for Marvin's exchange radial integral $N_{k-1}$ for the rest of the considered spin-other-orbit and spin-spin interaction is equal to zero, as will be shown below.

\section{Spin-Other-Orbit Interaction}

Concerning the matrix elements of the named operator, we start with the exchange terms which are the most complicated. The calculation is based on the general form formula (for any values of orbital moments of electrons) (8.41b) from [2]. The formula is very cumbersome and contains the summation on six parameters $k, K, x_{1}, x_{2}, x_{1}^{\prime}, x^{\prime}{ }_{2}$, where the parameter $k$ takes an unique value $k=6$ for radial integral $K^{\prime}{ }_{k} ; K=k \pm 1$; $x_{1}=0,1, \ldots, 2 l_{1} ; x_{2}=0,1, \ldots, 2 l_{2} ; x_{1}$ and $x_{2}$ take two values 0 and 1 in equal combinations. Furthermore, the cited formula is made up of the tensor products of unit orbital and spin operators $t_{12}^{x_{1} x_{2} 1}$ and $z_{12}^{x_{1} x_{2} 1}$, and also Racah's and Fano's coefficients, depending on the named parameters of summation.

There are two radial integrals here: the Marvin's exchange integral $N_{k-1}$ and $K_{k}^{\prime}$, related to Marvin's integral and characterizes only the spin-other-orbit interaction.

For alls-electron configurations the angular coefficient of $N_{k-1}$ tends to zero because of the factor a for $k=5,7$ in the cited formula or because the corresponding derived matrix elements of spherical functions operators equal zero. Thus what is left in the exchange part of matrix elements is the radial integral $K^{\prime}{ }_{k}$ with the angular coefficient of the following form:

$$
K_{k}^{\prime}=\frac{3 \sqrt{2}}{2 \sqrt{7 \cdot 13}}\left(z_{12}^{011}+z_{12}^{101}\right) t_{12}^{011}
$$

The phase factor, appearing according to the Wigner-Eckart theorem, here looks like:

(-1) power $\left(l_{1}+l_{2}+s_{1}+s_{2}-m_{l 1}-m_{l 2}-m_{s 1}-m_{s 2}\right)$.

Effecting the calculation in formula (10) with wave functions of independent momenta representation (1), we obtain following non null matrix elements:

$$
\begin{gathered}
\lambda_{1} \lambda_{1}=\lambda_{3} \lambda_{3}=-\frac{1}{2 \cdot 7 \cdot 13} K_{k}^{\prime} ; \\
\lambda_{1} \lambda_{2}=\lambda_{1} \lambda_{4}=\lambda_{2} \lambda_{3}=\lambda_{3} \lambda_{4}=\frac{\sqrt{42}}{2 \cdot 13 \cdot 14} K_{k}^{\prime}
\end{gathered}
$$

(the symmetric non diagonal matrix elements are not written).

During the permutation of electrons (the is configuration) matrix elements (11) have the opposite sign due to the factor $(-1)^{x_{2}}$ in the cited formula (8.41b) from [2].

For the hole $i^{25} s$ configuration the results are different. Namely the diagonal matrix elements are equal to zero, while the non-diagonal elements have the following form:

$$
\begin{gathered}
\lambda_{1} \lambda_{2}=\lambda_{3} \lambda_{4}=-\frac{\sqrt{42}}{4 \cdot 7 \cdot 13} K_{k}^{\prime} ; \\
\lambda_{1} \lambda_{4}=\lambda_{2} \lambda_{3}=\frac{\sqrt{42}}{4 \cdot 7 \cdot 13} K_{k}^{\prime}
\end{gathered}
$$

Let's effect the translation of matrix elements (11) and (12) to the LSJM representation with the help of the coefficients of transformation from table 1. For the si configuration we obtain the following exchange matrix elements of energy operator of spin-other-orbit interaction:

$$
\begin{array}{ll}
{ }^{3} I_{7}{ }^{3} I_{7}=\frac{3}{7 \cdot 13} K_{k}^{\prime} ; & { }^{3} I_{6}{ }^{3} I_{6}=-\frac{1}{13 \cdot 14} K_{k}^{\prime} ; \\
{ }^{3} I_{5}{ }^{3} I_{5}=-\frac{1}{26} K_{k}^{\prime} ; & { }^{1} I_{6}{ }^{1} I_{6}={ }^{3} I_{6}{ }^{1} I_{6}=0 .
\end{array}
$$

The matrix elements (13) of the is configuration have the opposite sign, while in hole $i^{25} s$ configuration the unit off-diagonal matrix elements are different from zero:

$$
{ }^{3} I_{6}{ }^{1} I_{6}=-\frac{\sqrt{42}}{7 \cdot 13} K_{k}^{\prime}
$$

In the monograph [2] the formula for the derived matrix elements of the considered operator in the LSJM representation was also given. The results of this independent calculation are the following:

$$
\left\|{ }^{3} I^{3} I\right\|=-\frac{3 \sqrt{13}}{13 \sqrt{7}} K_{k}^{\prime} ; \quad\left\|{ }^{3} I^{1} I\right\|=0 .
$$

The dependence of $(15)$ on the quantum number $\mathrm{J}$ is given by the following coefficients (see formula (8.13b) from [2]):

$$
\begin{aligned}
{ }^{3} I_{7}{ }^{3} I_{7}=-\frac{1}{\sqrt{7 \cdot 13}} ; & { }^{3} I_{6}{ }^{3} I_{6}=\frac{1}{6 \sqrt{7 \cdot 13}} \\
{ }^{3} I_{5}{ }^{3} I_{5}=\frac{\sqrt{7}}{6 \sqrt{13}} ; & { }^{3} I_{6}{ }^{1} I_{6}=-\frac{1}{\sqrt{3 \cdot 13}} .
\end{aligned}
$$

Multiplying the derived matrix elements (15) by the coefficient (16), we obtain the same matrix elements as in (13). It's impossible to calculate the holes $i^{25} s$ configuration in the LSJM representation, because here one has to change the sign of the orbital and spin projections of one $i$-electron, as was said earlier.

Now let's consider the direct matrix elements of energy operator of spin-other -orbit interaction. The general form formula (8.41b) from [2] (the independent momenta representation) after the corresponding mathematical transformation acquires the following form for configurations with $s$ - and $i$ - electrons: 
$|H|_{\text {пр. }}^{s o}=\left[(-1)^{l_{1}+l_{2}+s_{1}-m_{l 1}-m_{l 2}-m_{s 1}}(-12 \sqrt{7 \cdot 13}) t_{12}^{011} z_{1}^{1}\right.$.

$\delta\left(m_{s 2} m_{s 2}^{\prime}\right)+(-1)^{l_{1}+l_{2}+s_{2}-m_{l 1}-m_{l 2}-m_{s 2}}(-6 \sqrt{7 \cdot 13}) t_{12}^{011} z_{2}^{1}$.

$\left.\delta\left(m_{s 1} m_{s 1}^{\prime}\right)\right] S_{2}$

Here the direct Marvin's radial integral $M_{k-1}\left(n_{2} l_{2}, n_{1} l_{1}\right)$ (see (8.57) in [2]) for the unit value of the parameter $k=1$ is denoted by $S_{2}$. Let's pay attention to the fact that, in (17) the spin unit operators are one-electron type (compared with (10)). That is why the two terms have different phase factors and $\delta$-conditions.

The calculation of matrix elements by the formula (17) with the wave functions (1) gave the following results:

$$
\begin{aligned}
& \text { si: } \quad \lambda_{1} \lambda_{1}=\lambda_{3} \lambda_{3}=3 S_{2} ; \quad \lambda_{1} \lambda_{2}=\lambda_{3} \lambda_{4}=-\sqrt{42} S_{2} \text {; } \\
& \lambda_{1} \lambda_{4}=\lambda_{2} \lambda_{3}=-2 \sqrt{42} S_{2}
\end{aligned}
$$

$$
\text { is: } \begin{gathered}
\lambda_{1} \lambda_{1}=\lambda_{3} \lambda_{3}=3 S_{1} ; \quad \lambda_{1} \lambda_{2}=\lambda_{3} \lambda_{4}=-\sqrt{42} S_{1} ; \\
\lambda_{1} \lambda_{4}=\lambda_{2} \lambda_{3}=-2 \sqrt{42} S_{1} ;
\end{gathered}
$$

(the symmetric matrix elements are not written).

It seems that, angular coefficients in (18) and (19) are the same; in spite of the fact that in (17) the ranks of two-electron unit orbital operator $\left(t_{12}^{101}\right)$ change places. But as before, the upper rank of orbital operator 1 refers to $i$-electron, i.e. in the tensor product $t_{12}^{x_{1} x_{2} 1}$ nothing changes. The Marvin's radial integral $M_{k-1}\left(n_{2} l_{2}, n_{1} l_{1}\right)=S_{2}$ in the $s i$ configuration now becomes $M_{k-1}\left(n_{1} l_{1}, n_{2} l_{2}\right)=S_{1}$ in the $i s$ configuration, thus the lower indices in our notation indicate that this integral belongs to the $i$-electron (in is it's the first, while in si it's the second).

For the hole $i^{25} s$ configuration the change in (17) is the following: $t_{12}^{011}$ becomes $t_{12}^{101}$, consequently, the sign of this operator changes during the change of the sign of the orbital projection of the $i$ - electron. The sign of the phase factor in the first term of (17) changes. That is why for the hole $i^{25} s$ configuration we obtain the following matrix elements:

$$
\begin{aligned}
& \lambda_{1} \lambda_{1}=\lambda_{3} \lambda_{3}=-S_{1} ; \quad \lambda_{1} \lambda_{2}=\lambda_{3} \lambda_{4}=-\sqrt{42} S_{1} ; \\
& \lambda_{1} \lambda_{4}=\lambda_{2} \lambda_{3}=2 \sqrt{42} S_{1} .
\end{aligned}
$$

Comparing (19) and (20) we see that, the diagonal matrix elements in (20) are three times smaller and have the opposite sign as compared to the electronic is configuration (19). The matrix elements $\lambda_{1} \lambda_{4}=\lambda_{2} \lambda_{3}$ in is and $i^{25} s$ have opposite signs.

The translation of matrix elements (18)-(20) to the LSJM representation gives the following results:

$$
\begin{array}{ccc}
s i: & { }^{3} I_{7}{ }^{3} I_{7}=-18 S_{2} ; & { }^{3} I_{6}{ }^{3} I_{6}=3 S_{2} ; \\
& { }^{3} I_{5}{ }^{3} I_{5}=21 S_{2} ; & { }^{3} I_{6}{ }^{1} I_{6}=-\sqrt{42} S_{2} ; \\
i s: & { }^{3} I_{7}{ }^{3} I_{7}=-18 S_{1} ; & { }^{3} I_{6}{ }^{3} I_{6}=3 S_{1} ; \\
& { }^{3} I_{5}{ }^{3} I_{5}=21 S_{1} ; & { }^{3} I_{6}{ }^{1} I_{6}=-\sqrt{42} S_{1} ; \\
i^{25} s: & { }^{3} I_{7}{ }^{3} I_{7}=6 S_{1} ; & { }^{3} I_{6}{ }^{3} I_{6}=-S_{1} ; \\
& { }^{3} I_{5}{ }^{3} I_{5}=-7 S_{1} ; & { }^{3} I_{6}{ }^{1} I_{6}=-3 \sqrt{42} S_{1} .
\end{array}
$$

The comparison of matrix elements in the is and i25sconfiguration (21) show that, the last diagonal triplet matrix elements are thre e times smaller, while the off-diagonal matrix element ${ }^{3} I_{6}{ }^{1} I_{6}$ is three times greater and all with opposite sign as compared to the is configurations. It's the correct result (see [10]) and is one of the criteria of the validity of the obtained matrix elements.

The independent calculations of matrix elements of $s i$ and is configurations in LSJM-representation by formulae from [2] (8.43)-(8.50) completely coincide with results (21).

\section{Spin-Spin Interaction}

The energy operator of spin-spin interaction can be represented in the form of a scalar product of irreducible tensor operator of second order [2,5]: $H_{12}^{s s}=H_{12}^{220}$ (compare with $H_{12}^{s 0}=H_{12}^{110}$. Similar to the spin-other -orbit interaction, the angular coefficient in the Marvin's radial exchange integral $N_{k-1}$ tends to zero due to stated matrix elements of operator of spherical functions (see (7.31b) in [2]). Let's explain this. The parameter of summation $\mathrm{k}$ in the cited formula takes two values: $\mathrm{k}=5$ and $\mathrm{k}=7$, while the stated matrix elements of operator of spherical functions are written in a product form as:

$$
\left(l_{1}\left\|C^{k+1}\right\| l_{2}\right)\left(l_{2}\left\|C^{k-1}\right\| l_{1}\right) .
$$

It is clear that, when $\mathrm{k}=5$ the second factor is equal to zero, while when $\mathrm{k}=7$ the first factor nullifies for the $s$-electron configuration (in our case $l_{1}=0 ; l_{2}=6$ ).

For the direct matrix elements of the considered operator from formula (7.31a) in [2] after corresponding mathematical transformation we have:

$$
|H|_{\text {пр. }}^{S S}=\frac{12 \cdot \sqrt{3 \cdot 7 \cdot 13}}{\sqrt{5 \cdot 11}} t_{12}^{022} \cdot z_{12}^{112} S_{2} .
$$

Here $S_{2}$ is also the direct Marvin's radial integral $M_{k-1}$ when $\mathrm{k}=0$, in the spin-other-orbit interaction (see formula (17) and comments therein). The tensor product of unit orbital $t_{12}^{022}$ and spin $z_{12}^{112}$ operators are transformed according to formula (14.45) from [5] in the direct products, and to each of operators $t_{1}^{0}, t_{2}^{2}, z_{1}^{1}, z_{2}^{1}$ one uses the Wigner-Eckart theorem. According to the later, in (23) appears a phase factor, equal to (-1) power $\left(l_{1}+l_{2}+s_{1}+\right.$ $\left.s_{2}-m_{l 1}-m_{l 2}-m_{s 1}--m_{s 2}\right)$, while the corresponding 
matrix elements $\left(l_{1} l_{2}\left\|t^{02}\right\| l_{1} l_{2}\right)=\left(l_{1}\left\|t^{0}\right\| l_{1} l\right)\left(l_{2}\left\|t^{2}\right\| l_{2}\right) \quad$ are equal to unity (also for spin operators).

From (23) for the si configuration we obtained the following matrix elements in independent moments representation:

$$
\begin{aligned}
& \lambda_{1} \lambda_{1}=\lambda_{3} \lambda_{3}=-\frac{26}{55} S_{2} ; \lambda_{2} \lambda_{2}=\lambda_{4} \lambda_{4}=\lambda_{2} \lambda_{4}=\frac{28}{55} S_{2} ; \\
& \lambda_{1} \lambda_{2}=\lambda_{3} \lambda_{4}=\lambda_{1} \lambda_{4}=\lambda_{2} \lambda_{3}=-\frac{\sqrt{42}}{55} S_{2} ; \lambda_{1} \lambda_{3}=\frac{84}{55} S_{2}
\end{aligned}
$$

For the is configuration we have the same angular coefficients, as in (24), but already in radial integral $S_{1}$ ((19)). In the $\mathrm{i}^{25} \mathrm{~s}$ hole configuration the matrix elements with the change $S_{2} \rightarrow S_{1}$ have the opposite sign due to the phase factor.

Further transforming the matrix elements (24) to the LSJM representation with the help of coefficients of transformation from table1, we have:

$$
{ }^{3} I_{7}{ }^{3} I_{7}=\frac{4}{5} S_{2} ; \quad{ }^{3} I_{6}{ }^{3} I_{6}=-2 S_{2} ; \quad{ }^{3} I_{5}{ }^{3} I_{5}=\frac{14}{11} S_{2} .
$$

The off-diagonal matrix element ${ }^{3} I_{6}{ }^{1} I_{6}$ here is equal to zero, in contrast with the spin-other-orbit interaction (see (21)).

The comments to formula (24) are true for the is and $i^{25}$ configurations.

Let's calculate the matrix elements of the considered operator in LSJM representation. The derived matrix elements of the considered operator have the following form (the formula is derived from [2]):

$$
\begin{gathered}
\left(n_{1} n_{2} l_{1} l_{2} L S\left\|H_{12}^{s s}\right\| n_{1} n_{2} l_{1} l_{2} L^{\prime} S^{\prime}\right)=\delta(S, 1) \delta\left(S^{\prime}, 1\right) \times \\
\times \sum_{k}\left[\begin{array}{l}
m_{k-1}\left(l_{1} l_{2} L L^{\prime}\right) \cdot M_{k-1}\left(n_{1} l_{1}, n_{2} l_{2}\right)+ \\
\tilde{m}_{k-1}\left(l_{1} l_{2} L L^{\prime}\right) \cdot M_{k-1}\left(n_{2} l_{2}, n_{1} l_{1}\right)+ \\
n_{k-1}\left(l_{1} l_{2} L L^{\prime}\right) \cdot N_{k-1}\left(n_{1} l_{1}, n_{2} l_{2}\right)
\end{array}\right]
\end{gathered}
$$

Here the parameter $k$ takes a unique value $k=1$. In this expression there is the $\delta$-condition on spin, from which it follows that only triplet matrix elements $\left(S=S^{\prime}=1\right)$ are non-null as is the case with diagonal elements (see also $[11,12])$. Furthermore, the angular coefficient for the Marvin's radial exchange integral $N_{k-1}$ tends to zero due to the product (see (7.38) in [1]). The angular coefficient for Marvin's direct integral in the first term of (26) also tends to zero, as in the expression (7.36) from [2] the derived matrix element $\left(0\left\|C^{2}\right\| 0\right)=0$.

Thus, matrix elements of the energy operator of the spin-spin interaction are represented by the second term in (26). Let's write his angular coefficient (formula (7.36) from [2]):

$$
\begin{aligned}
& \tilde{m}_{k-1}\left(l_{1} l_{2} L L^{\prime}\right)= \\
& =A\left(l_{1}\left\|C^{k-1}\right\| l_{1}\right)\left(l_{2}\left\|C^{k+1}\right\| l_{2}\right)\left\{\begin{array}{lll}
l_{1} & l_{1} & k-1 \\
l_{2} & l_{2} & k+1 \\
L & L^{\prime} & 2
\end{array}\right\}
\end{aligned}
$$

Here the factor $A=\sqrt{5(2 k+3)^{(5)}(2 L+1)\left(2 L^{\prime}+1\right)}=$ $=130 \sqrt{6}$ for the considered si configuration.

The calculation by the formula (27) gave the following result:

$$
\widetilde{m}_{k-1}=\frac{4 \sqrt{3 \cdot 7 \cdot 13}}{\sqrt{11}} .
$$

All the matrix elements of the considered operator, taking in to account the quantum number $\mathrm{J}$ (further coefficient D), are determined by the formula (derived from [2]):

$$
\left|H^{220}\right|=(-1)^{L+S+J}\left(L S\left\|H^{s S}\right\| L^{\prime} S^{\prime}\right)\left\{\begin{array}{ccc}
L & S & J \\
S^{\prime} & L^{\prime} & 2
\end{array}\right\} .
$$

The coefficients D, calculated by this formula, have the following values:

$$
\begin{aligned}
& { }^{3} I_{7}{ }^{3} I_{7}=\frac{\sqrt{11}}{5 \sqrt{3 \cdot 7 \cdot 13}} ; \quad{ }^{3} I_{6}{ }^{3} I_{6}=-\frac{\sqrt{11}}{2 \sqrt{3 \cdot 7 \cdot 13}} ; \\
& { }^{3} I_{5}{ }^{3} I_{5}=\frac{\sqrt{7}}{2 \sqrt{3 \cdot 11 \cdot 13}} .
\end{aligned}
$$

It's not difficult to verify that, multiplying (28) by the coefficients (30), we have the same results $(25)$, as in the independent moments representation with the translation to the LSJM representation, as was required.

\section{Conclusion}

The energy operator interaction matrix has been built for the orbit-orbit electrostatic, the spin-other-orbit, the spin-spin interactions for $s i, i s, i^{25} s$ configurations. The calculation of matrix elements was done in the independent moments representation with the further translation to the LSJM representation for the si and is electronic configurations. The results of the calculation by both representations coincided, thus this testifies to the accuracy of matrix elements of electronic configurations. For the holes $i^{25} s$ configurations there was no need to effect new calculations, it was sufficient to take into account the change of the sign of orbital and spin projections of $i$-holes (the quasi-full shell), in the matrix elements of the is configuration. It concerned the phase factor, the $\delta$ -condition on spins projection and the orbital operator of odd rank $t_{1}^{1}$ (the sign of the corresponding Wigner $3 \mathrm{j}$-symbol).

The matrix energy operator of the above listed interactions is destined for the subsequent numerical calculations of the fine structure parameter, the coefficient of the intermediate constraints, the gyromagnetic relations, and also for the study of Zeeman splitting. 


\section{References}

[1] NIST Atomic Spectra Database Levels Data. 2008.

[2] Yutsis A.P., Savukinas A.Yu. Mathematical basis of atom theory (in Russian) Vilnius, 1973, 479 P.

[3] Anisimova G.P., Kapel'kina E.L. "Orbit-orbit interaction in two electronic matrix energy operator of configurations with $p$ and $s$ electrons". Optics and spectroscopy. 1999. T. 87, №6, pp.885-892.

[4] Anisimova G.P., Efremova E.A., Tsygankova G.A., Tsygankov M.A. "Calculation of fine and Zeeman structure of configuration $2 n p n d+2 p(n+1)$ s of a carbon atom by semi empirical method," Vestnik of Saint-Petersburg State University, ser.4, issue 36, 2007, pp. 49-60.

[5] Sobelman I.I. Introduction to the theory of atomic specters. (in Russian) M. 1963, 640 p.

[6] Varshalovich D.A., Moscalev A.H., Hersonski V.K. Quantum theory of angular momenta. (in Russian) L., 1975, 439 P.

[7] Kondon E., Shortly G. Theory of atomics specters. (in Russian) M. 1949, 440 p.

[8] Anisimova G.P., Semenov R.I., Tuchkin V.I. Optics and spectroscopy 1996. T. 80. N4 PP.544-556.

[9] Anisimova G.P., Semenov R.I., Tuchkin V.I. Optics and spectroscopy 1996. T. 76. N5 PP. 720-725.

[10] Anisimova G.P., Kapel'kina E.L. "Spin-other-orbit interaction in two electronic matrix energy operator of configurations with $p$ and $s$ electrons. Direct terms". Optics and spectroscopy. 1998.T. 84,n3, pp.364-368.

[11] Anisimova G.P., Semenov R.I., Tuchkin V.I. Optics and spectroscopy 1994. T. 77. N5 PP. 695-699.

[12] Anisimova G.P., Kapel'kina E.L. "The account of spin-spin interaction in the energy matrices of the two electrons configurations with $p$ and $s$ electrons". Optics and spectroscopy. 1999.T. 87,n1, pp.15-21. 